\title{
SIMULTANEOUS MINIMAL MODELS OF HOMOGENEOUS TORIC DEFORMATIONS
}

\author{
DAISUKE MATSUSHita
}

\begin{abstract}
Every flat family of Du Val singularities admits a simultaneous minimal resolution after a finite base change. We investigate a flat family of isolated Gorenstein toric singularities and prove that there exists a simultaneous partial resolution.
\end{abstract}

\section{Introduction}

For a flat family of surfaces $f: X \rightarrow S$, a birational morphism $\tau: \tilde{X} \rightarrow X$ is said to be simultaneous minimal resolution if $\tau$ satisfies the following two conditions:

(1) $f \circ \tau$ is a flat morphism.

(2) $\tilde{X}_{s}:=(f \circ \tau)^{-1}(s)(s \in S)$ is the minimal resolution of $X_{s}$. Let $f: X \rightarrow S$ be a flat morphism whose central fibre $f^{-1}(0)$ has only Du Val singularities. Brieskorn [3, 4] and Tyurina [11] proved that there exists an open set $0 \in U,(U \subset S)$ and a finite surjective morphism $U^{\prime} \rightarrow U$ such that a flat morphism $f^{\prime}: X \times_{U} U^{\prime} \rightarrow U^{\prime}$ admits a simultaneous minimal resolution. We consider an analogous problem for a flat family of isolated Gorenstein toric singularities. According to the Minimal Model Theory, it is natural to consider an existence of "simultaneous terminalization" for a flat family of higher dimensional singularities.

Definition 1. Let $f: X \rightarrow S$ be a flat morphism. It is said that $f$ admits a simultaneous terminalization if there exists a birational morphism $\tau: \tilde{X} \rightarrow X$ which satisfies the following conditions:

(1) $f \circ \tau$ is a flat morphism.

(2) $\tilde{X}_{s}:=(f \circ \tau)^{-1}(s)(s \in S)$ has only terminal singularities.

(3) $K_{\tilde{X}_{s}}$ is $\tau$-nef.

By [2, Theorem 8.1], an $n$-dimensional isolated toric singularity is rigid if $n \geq 4$ or it is not Gorenstein. Hence we investigate a flat family of 3-dimensional isolated Gorenstein toric singularities. Our result is the following:

1991 Mathematics Subject Classification: Primary 14E30; Secondary 14B07, 14M25.

Received April 16, 2001; revised July 9, 2001. 
THEOREM 1.1. Let $f: X \rightarrow S$ be a flat morphism such that the central fibre $f^{-1}(0)$ has only 3-dimensional isolated Gorenstein toric singularities and the base space $S$ is reduced. Then there exist an open neighbourhood $0 \in U,(U \subset S)$ and a birational morphism $\tau: \tilde{X} \rightarrow X \times{ }_{S} U$ which satisfy the following conditions:

(1) $f \circ \tau: \tilde{X} \rightarrow U$ is a flat morphism.

(2) The fibre $\tilde{X}_{s}$ has only hypersurface singularities in cyclic quotient space. Moreover those singularities are defined by

$$
\{x y-z w=0\} \subset C^{4} / G, \quad G \cong Z / n Z,
$$

where the action of $G$ is given by

$$
(x, y, z, w) \rightarrow\left(\zeta x, \zeta^{-1} y, \zeta^{a} z, \zeta^{-a} w\right),
$$

( $\zeta$ is an $n$-th root of unity).

(3) $K_{\tilde{X}_{s}}$ is $\tau$-nef.

Remark 1. The singularity of Theorem 1.1 (3) is not terminal singularity if $G$ is not trivial. We construct an example of a flat family of isolated Gorenstein singularity which admits no simultaneous terminalization even if after finite base change. Please see Remark 4 in section 3.

This note is organised as follows: We recall the definition of homogeneous toric deformation according to $\mathrm{K}$. Altmann in section 2 . Theorem 1 is proved in section 3 .

\section{Homogeneous toric deformation}

The following definition of homogeneous toric deformation is introduced by K. Altmann in [1, Definition 3.1].

Definition 2. A flat morphism $f: X \rightarrow C^{m}$ is called a homogeneous toric deformation if the following conditions are satisfied:

(1) $X:=\operatorname{Spec} C\left[\sigma^{\vee} \cap M\right]$ is an affine toric variety.

(2) $f$ is defined by $m$ equations $x^{r_{i}}-x^{r_{0}}=t_{i}(1 \leq i \leq m)$, where $r_{i} \in \sigma^{\vee} \cap M$ and $t_{1}, \ldots, t_{m}$ are coordinates of $\boldsymbol{C}^{m}$.

(3) Let $L:=\bigoplus_{i=1}^{i=m} \boldsymbol{Z}\left(r_{i}-r_{0}\right)$ be the sublattice of $M$. The central fibre $Y:=f^{-1}(0, \ldots, 0)$ is isomorphic to Spec $C\left[\bar{\sigma}^{\vee} \cap \bar{M}\right]$ where $\bar{\sigma}=\sigma \cap L^{\perp}$ and $\bar{M}:=M / L$.

(4) $i: Y \rightarrow X$ sends the closed orbit in $Y$ isomorphically onto the closed orbit in $X$.

In this note, we consider a homogeneous toric deformation with some additional conditions:

DeFinition 3. We call homogeneous toric deformation $f: X \rightarrow \boldsymbol{C}^{m}$ a Gorenstein homogeneous toric deformation if it satisfies the following two conditions: 
(1) $Y$ has only Gorenstein singularities.

(2) Kodaira-Spencer map $\boldsymbol{C}^{m} \rightarrow T_{Y}^{1}$ is nontrivial.

Remark 2. We list some examples of Gorenstein homogeneous toric deformation.

(1) The simplest example is $f: \boldsymbol{C}^{2} \rightarrow \boldsymbol{C}$ defined by $x-y=t$.

(2) Let $g: \mathscr{X} \rightarrow S$ be a versal deformation space of Du Val singularity of type $A_{n}$. The space $\mathscr{X}$ is defined by the equation

$$
\mathscr{X}=\left(x y+z^{n+1}+t_{1} z^{n-1}+\cdots+t_{n-1} z+t_{n}=0\right)
$$

in $\boldsymbol{C}^{n+3}$ and $g$ is the projection. Let $\alpha_{i}(0 \leq i \leq n)$ be the $i+1$-th elementary symmetric polynomials of $(n+1)$-variables and $H$ a hyperplane in $\boldsymbol{C}^{n+1}$ defined by $\sum_{i=0}^{n} s_{i}=0$, where $s_{0}, \ldots, s_{n}$ are coordinates of $\boldsymbol{C}^{n+1}$. We take a base change by $\alpha: H \rightarrow C^{n}\left(\alpha^{*} t_{i}=\alpha_{i}\left(s_{0}, \ldots, s_{n}\right)\right)$.

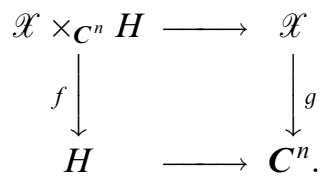

Then $\mathscr{X} \times{ }_{C^{n}} H$ can be described

$$
\left\{x y+\prod_{i=0}^{n}\left(z+s_{i}\right), \sum_{i=0}^{n} s_{i}=0\right\} \subset C^{n+4} .
$$

Using new coordinates $z_{i}:=z+s_{i}, \mathscr{X} \times{ }_{C^{n}} H$ is written as

$$
\mathscr{X} \times \boldsymbol{C}^{n} H=\left(x y+\prod_{i=0}^{n} z_{i}=0\right) \subset \boldsymbol{C}^{n+3}
$$

and $f=\left(z_{1}-z_{0}, \ldots, z_{n}-z_{0}\right)$. Thus $f: \mathscr{X} \times{ }_{C^{n}} H \rightarrow H$ is a Gorenstein homogeneous toric deformation.

(3) Let $g: \mathscr{X} \rightarrow \mathscr{M}$ be a versal deformation space of an $n$-dimensional $(n \geq 3)$ isolated Gorenstein toric singularity. We denote by $\mathscr{S}$ an irreducible component of $\mathscr{M}$ and by $\mathscr{S}_{\text {red }}$ its reduced structure. By $[2$, Theorem 8.1], the base change $f: \mathscr{X}_{\text {red }}:=\mathscr{X} \times \mathscr{S} \mathscr{S}_{\text {red }} \rightarrow \mathscr{S}_{\text {red }}$ is a Gorenstein homogeneous toric deformation.

\section{Simultaneous minimal model of Gorenstein homogeneous toric deformation}

Theorem 1.1 is obtained as a corollary of the following theorem.

TheOREM 3.1. Let $f: X:=\operatorname{Spec} C\left[\sigma^{\vee} \cap M\right] \rightarrow C^{m}$ be a Gorenstein homogeneous toric deformation and $\tau: \tilde{X} \rightarrow X$ a toric minimal model of $X$. Assume that $\operatorname{dim} X=n+m$. Then 
(1) $f \circ \tau: \tilde{X} \rightarrow C^{m}$ is a flat morphism,

(2) $K_{\tilde{X}_{t}}$ is $\tau$-nef,

(3) $\tilde{X}_{t}$ has only hypersurface singularities in a quotient space. Moreover these singularities are defined by

$$
\left(F_{i}-F_{0}=0\right) \subset C^{n+m} / G, \quad(1 \leq i \leq m)
$$

where

(a) $G$ is an abelian group which acts on $\boldsymbol{C}^{n+m}$ diagonally,

(b) $C^{n+m} / G$ has only Gorenstein terminal singularities,

(c) Each $F_{i}$ is written as

$$
\begin{gathered}
F_{i}=\prod_{j=p_{i}+1}^{p_{i+1}} x_{j} \quad(0 \leq i \leq m) \\
0=p_{0}<p_{1}<p_{2}<\cdots<p_{m}<p_{m+1}=n+m
\end{gathered}
$$

where $x_{j}$ is the $j$-th coordinate of $C^{n+m}$. Moreover $F_{i}$ are invariant monomials under the action of $G$.

Remark 3. If $\operatorname{dim} X=2+m$ (i.e. Every fibre of $f$ is 2-dimensional), then $F_{i},(1 \leq i \leq m)$ is written as

$$
F_{i}=x_{i+1} \quad(0 \leq i \leq m-1), \quad F_{m}=x_{m} x_{m+1}
$$

by changing indices if necessary. Because $F_{i}$ are invariant monomials under the action of $G$, the action of each element of $g \in G$ is nontrivial only on coordinates $x_{m}$ and $x_{m+1}$. Since $C^{2+m} / G$ has only Gorenstein terminal singularities, the action of $G$ must be trivial. Thus each fibre of $f \circ \tau$ is smooth and $\tau$ gives a simultaneous resolution of $f$.

Proof of Theorem 1.1. Since $S$ is reduced, there exists an open set $0 \in U$, $(U \subset S)$ which satisfies the following commutative diagram:

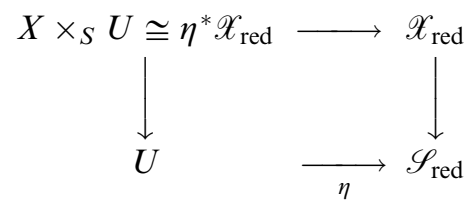

where $\eta$ is an open immersion and $\mathscr{X}_{\text {red }} \rightarrow \mathscr{S}_{\text {red }}$ is the restriction of a versal deformation space to some irreducible component with its reduced structure. For Theorem 1.1, it is enough to prove that there exists a birational morphism $\tau: \widetilde{\mathscr{X}_{\text {red }}} \rightarrow \mathscr{X}_{\text {red }}$ which satisfies the assertions of Theorem 1.1. By [2, Theorem 8.1], we describe $\mathscr{X}_{\text {red }} \rightarrow \mathscr{S}_{\text {red }}$ as a Gorenstein homogeneous toric deformation. Then by Theorem 3.1, there exists a birational morphism $\widetilde{\mathscr{X}_{\text {red }}} \rightarrow \mathscr{X}_{\text {red }}$ which satisfies assertions (1) and (2) of Theorem 1.1. We check the assertion (3). Because $\operatorname{dim} \widetilde{\mathscr{X}_{\text {red }}}=\operatorname{dim} \mathscr{S}_{\text {red }}+3, F_{i}$ is written as

$$
F_{i}=x_{i}(0 \leq i \leq m-2), \quad F_{m-1}=x_{m-1} x_{m}, \quad F_{m}=x_{m+1} x_{m+2}
$$


or

$$
F_{i}=x_{i}(0 \leq i \leq m-1), \quad F_{m}=x_{m} x_{m+1} x_{m+2} .
$$

Each $F_{i}$ are invariant monomials under the action of $G$. Hence, in the latter case, singularities of a fibre is isomorphic to $C^{3} / G$. There exists no 3-dimensional Gorenstein quotient terminal singularities. Thus $G$ is trivial. Therefore the central fibre has only the following singularities:

$$
\left\{x_{m-1} x_{m}-x_{m+1} x_{m+2}=0\right\} \subset C^{4} / G .
$$

Again there exists no 3-dimensional Gorenstein quotient terminal singularities. Hence $C^{4} / G$ has only isolated singularities. The proof of Theorem 1.1 is completed by the classification of 4-dimensional isolated Gorenstein toric singularities [8, Theorem 2.4].

Proof of Theorem 3.1. By [1, Theorem 3.5, Remark 3.6], the construction of $\sigma$ is as follows:

(1) $\sigma$ is defined by $\sigma=\boldsymbol{R}_{\geq 0} P$, where $P$ is an $(n+m-1)$-dimensional polygon given by

$$
P:=\operatorname{Conv}\left(\bigcup_{i=0}^{m} R_{i} \times e_{i}\right) .
$$

Note that $R_{i}(0 \leq i \leq m)$ are integral polytopes in $\boldsymbol{R}^{n-1}$ and

$$
R_{i} \times e_{i}:=\left\{\left(x_{1}, \ldots, x_{n-1}, 0, \ldots, 1, \ldots, 0\right) \in \boldsymbol{R}^{n+m} \mid\left(x_{1}, \ldots, x_{n-1}\right) \in R_{i}\right\} .
$$

(2) $f$ is defined by $\left(x^{r_{i}}-x^{r_{0}}\right)(1 \leq i \leq m)$, where $r_{i}: N_{\boldsymbol{R}}=\boldsymbol{R}^{n+m} \rightarrow \boldsymbol{R}$ is the $(n+i)$-th projection.

Thus, all primitive one dimensional generators of $\sigma$ are contained in the hyperplane in $N_{\boldsymbol{R}}$ defined by $r_{0}+\cdots+r_{m}=1$. By [9, Theorem 0.2], there exists a toric minimal model $\tilde{X}$. Let $\sigma=\bigcup \sigma_{\lambda}$ be the corresponding cone decomposition. By [9, Definition 1.11], these cones satisfy the following three conditions:

(1) $\sigma_{\lambda}$ is a simplex.

(2) One dimensional primitive generators $k_{1}, \ldots, k_{n+m}$ of $\sigma_{\lambda}$ are contained in the hypersurface defined by $r_{0}+\cdots+r_{m}=1$.

(3) The polytope

$$
\Delta_{\lambda}:=\sum_{j=0}^{n+m} \alpha_{j} k_{j}, \quad \sum_{j=0}^{n+m} \alpha_{j} \leq 1, \quad \alpha_{j} \geq 0
$$

contains no lattice points except its vertices.

Let $X_{\lambda}:=\operatorname{Spec} C\left[\sigma_{\lambda}^{\vee} \cap M\right]$ and let $k_{j}^{\vee}(1 \leq j \leq n+m)$ be the dual vectors of $k_{j}$. By (1), $X_{\lambda}$ can be written as follows:

$$
X_{\lambda} \cong C^{n+m} / G
$$

where $G:=N / \bigoplus_{j=1}^{n+m} \boldsymbol{Z} k_{j}$ and the action of $G$ is diagonal. Because each $k_{j}$ are contained in the hypersurface defined by $r_{0}+\cdots+r_{m}=1$ and $\left\langle r_{i}, k_{j}\right\rangle \geq 0$ $\left(r_{i} \in \sigma^{\vee}\right)$, 


$$
\begin{cases}\left\langle r_{i}, k_{j}\right\rangle=1 & \text { for } p_{i}<j \leq p_{i+1} \\ \left\langle r_{i}, k_{j}\right\rangle=0 & \text { other } j\end{cases}
$$

where $0=p_{0}<p_{1}<p_{2}<\cdots<p_{m}<p_{m+1}=n+m$. Thus $x^{r_{i}}$ is written as

$$
x^{r_{i}}=\prod_{j=p_{i}+1}^{p_{i+1}} x_{j}
$$

where $x_{j}=x^{k_{j}^{\vee}}$ is the $j$-th coordinate of $C^{n+m}$. The monomials $x^{r_{i}}$ are invariant under the action of $G$, because $r_{i} \in \sigma_{\lambda}^{\vee} \cap M$. Thus if we set $F_{i}=x^{r_{i}}$, the proof of Theorem 3.1 is completed.

Remark 4. There exists an example of a flat family of isolated Gorenstein toric singularity which has no simultaneous terminalization even after finite base change.

LEMMA 3.2. Let $Y$ be a hypersurface singularity in a cyclic quotient space defined by

$$
\left\{x_{1} x_{2}-x_{3} x_{4}=0\right\} \subset C^{4} / G, \quad G \cong Z / l Z .
$$

where the action of $G$ given by

$$
\begin{gathered}
\left(x_{1}, \ldots, x_{4}\right) \rightarrow\left(\zeta^{a_{1}} x_{1}, \ldots, \zeta^{a_{4}} x_{n+1}\right), \quad\left(0<a_{i}<l\right) \\
a_{1}+a_{2} \equiv a_{3}+a_{4} \equiv 0 \quad(\bmod l) .
\end{gathered}
$$

Note that $\zeta$ is a primitive l-root of unity and $a_{i}$ 's are coprime. Let $X$ be the subvariety $C^{4} / G \times C$ defined by

$$
x_{1} x_{2}-x_{3} x_{4}=t
$$

and $f: X \rightarrow C$ the projection. Then $Y$ has only isolated Gorenstein toric singularities and $f$ admits no simultaneous terminalization even after any finite base change.

Proof. It is easy to see that $Y$ has only isolated toric singularities. Since the residue form

$$
\operatorname{Res} \frac{d x_{1} \wedge d x_{2} \wedge d x_{3} \wedge d x_{4}}{x_{1} x_{2}-x_{3} x_{4}}
$$

is $G$-equivariant, $Y$ has only isolated Gorenstein toric singularities. Because

$$
\sum_{i=1}^{4} a_{i} \geq 2 l
$$

$C^{4} / G$ has only Gorenstein terminal singularities. We derive a contradiction assuming that there exists a simultaneous terminalization after some finite base change. Let $Z$ be the subvariety $C^{4} / G \times C$ defined by

$$
x_{1} x_{2}-x_{3} x_{4}=t^{m} \text {. }
$$


From the assumption, there exists a simultaneous terminalization $\tau: \mathscr{X} \rightarrow Z$. Let $Z^{\prime}$ be the subvariety in $C^{5}$ defined by

$$
x_{1} x_{2}-x_{3} x_{4}=t^{m} \text {. }
$$

Then there exists a finite morphism $Z^{\prime} \rightarrow Z$. Since $Z^{\prime}$ has only hypersurface singularities whose singular locus has codimension four, it is $\boldsymbol{Q}$-factorial by [7, XI.3.13]. By [6, Lemma 5.16], $Z$ is again $\boldsymbol{Q}$-factorial. Because a general fibre of $f: X \rightarrow \boldsymbol{C}$ is smooth, the codimension of exceptional set of $\tau$ is greater than two. That contradicts to [5, VI 1.5 Theorem].

\section{REFERENCES}

[1] K. Altmann, Minkowski sums and homogeneous deformations of toric varieties, Tôhoku Math. J. (2), 47 (1995), 151-184.

[2] K. Altmann, The versal deformation of an isolated toric Gorenstein singularity, Invent. Math., 128 (1997), 443-479.

[3] E. BRIESKorn, Über die Auflösung gewisser Singularitäten von holomorphen Abbildungen, Math. Ann., 166 (1966), 76-102.

[4] E. BriesKorn, Die Auflösung der rationalen Singularitäten holomorpher Abbildungen, Math. Ann., 178 (1968), 255-270.

[ 5 ] J. Kollár, Rational Curves on Algebraic Varieties, Ergeb. Math. Grenzgeb. (3) 32, SpringerVerlag, Berlin, 1996.

[6] J. Kollár and S. Mori, Birational Geometry of Algebraic Varieties, Cambridge Tracts in Math. 134, Cambridge University Press, Cambridge, 1998.

[ 7 ] A. Grothendieck, Cohomologie Locale des Faisceaux Cohérents et Théorèmes de Lefschetz Locaux et Globaux (SGA2), North-Holland, Amsterdam, 1968.

[ 8 ] D. R. Morrison and G. Stevens, Terminal quotient singularities in dimensions three and four, Proc. Amer. Math. Soc., 90 (1984), 15-20.

[9] M. ReID, Decomposition of toric morphisms, Arithmetic and Geometry Volume II (M. Artin and J. Tate eds.), Progress in Math. 36, Birkhäuser, Boston, 1983, 395-418.

[10] M. ReID, Young person's guide to canonical singularities, Algebraic Geometry Bowdoin 1985 (S. J. Bloch ed.), Proc. Sympos. Pure Math. 46-1, AMS, Providence, 1987, 345-414.

[11] G. N. Tyurina, Resolution of singularities of plane deformations of double rational points, Functional Anal. Appl., 4 (1970), 68-73.

Division of Mathematics

Graduate School of Science

HOKKAIDO UNIVERSITY

SAPPORO, 060-0810 JAPAN

e-mail: matusita@math.sci.hokudai.ac.jp 